supported, is introduced in to the hollow central part of the mould, the molten glass being prevented by a
button from entering the latter. The lip of the bottle is shaped first, and the mould is then quickly reversed, When the mass has descended to the length required the halves of the mould are closed on it, a current of air from the pump is turned on, and the bottle quickly
blown into the shape of the mould. A different mould blown into the shape of the mould. A differe
is, of course, required for each kind of bottle.

The principal improvement which it is proposed to introd uce into the machine now at work is to fix a volving stand, turning round as quickly as possible, with the glass metal, another working the air pump and closing the moulds, and a third taking out the finished bottles.

The first result of the introduction of the perfected machines will be to diminish the number of hands em-
ployed; but as a large increase of orders is anticipated, owing to the impossibility of foreign competition, which now supplies this country with about 16,000 bottles per day, or two and a half times as much as we ourselves manufacture, it is thought that the growth
of business will quickly necessitate the employment of of business will quickly necessitate the employment of even a larger number of hands than are now engaged. ing capacity of Messrs. Sykes, Macvay \& Co.'s works, if sixty machines were set at work (one for each "hole" now existing), would be increased tenfold, or from 5,400 to 54,000 dozen bottles per day, while, instead of 300 ,
only 180 hands would be required, and the cost of labor would be reduced from $3 \mathrm{~s}$. $10 \mathrm{~d}$. to $3 \mathrm{~d}$. per gross.
The exploiters of the patent propose to allow other firms to use the machine upon payment of a royalty, works, and duly numbered and registered.

Mr. Ashley's partner in the patent, in the course of conversation, told our representative that, when they first asked permission from bottle manufacturers to
put the invention to a practical test, they were everywhere received with suspicion, and found all works proper supply of molten glass without the employment of expensive fireclay pots, the erection of ovens and was resolved to use a mixture of molten shellac and resin instead of the glass metal. The inventor then constructed an improvised machine in an empty blackThese were shown to Messrs. Sykes, Macvay \& Co., who
thereupon decided to take the matter up.

THE POLAR PLANIMETER-ITS THEORY AND I'I'S USE*

By E. A. Gieseler, C.E.

By means of this instrument the area of any regular or irregular plane figure can be found by running the lowing the writer has attempted to give the theory of erally given.

and IT, rotating consists of a system of two bars, $F$

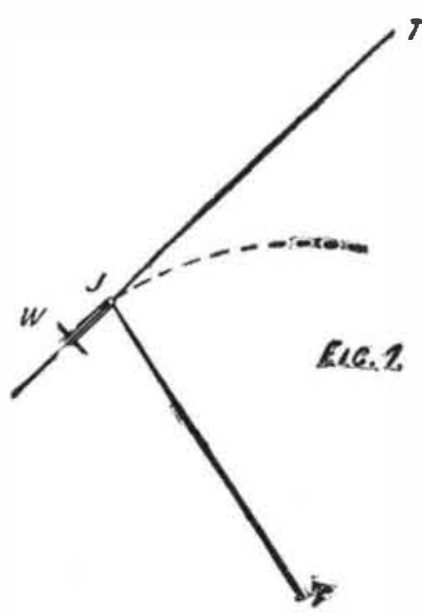

One of them. IF, which we will designate as the polar arm, is jointed at I to a sleeve, in which slides the attached to it, there is an axle revolving in bearings
and carrying a small wheel. W. The bar, $\mathrm{I}$, which we
will designate as the movable arm, can be shifted and clamped in any desired position in the sleeve; the disextreme end for the purpose of following the lines of its setting depends the value of the unit in which are expressed the results obtained by the instrument. When the apparatus is put up for use, it rests on the
plane of the drawing with three points, viz., the tracer, $T$, one point of the circumference of the wheel, $W$, and the fulcrum or pole, $F$, which latter consists of a ball
joint held in place by a heavy weight, and around clear that with the tracer of this instrument the contour of any area can be circumscribed, provided that the size of the same does not exceed the limit of the
apparatus, and further, that the position of the fulcrum has been properly chosen. This can be selected either inside or outside of the figure the area of which 1. When thef ulcrum is outside of the are - In Figs. 2 and 3, A represents the figure the area of which is to be found; the instrument, with its fulcrum outside, is shown in various positions, the sleeve carrying the
wheel having been left out so as not to unnecessarily complicate the diagrams. at us suppose the starting point of the tracer to be ed on its path around it, in the direction of the arrow its new positions will be to the right of the extended
line I T.
Now the journey of the tracer around the entire contour may be considered as consisting of two distinct
parts. First, the part in which the tracer travels rom its first, the above described extreme left position, the extreme right position, from which any further movement will bring it to the left of the then position, the tracer travels from the extreme right position along the lower part of the contour until it arrives once more the starting point. The first part of the tracer's Fig. 3 ; in both diagrams two. 2 , the second part in

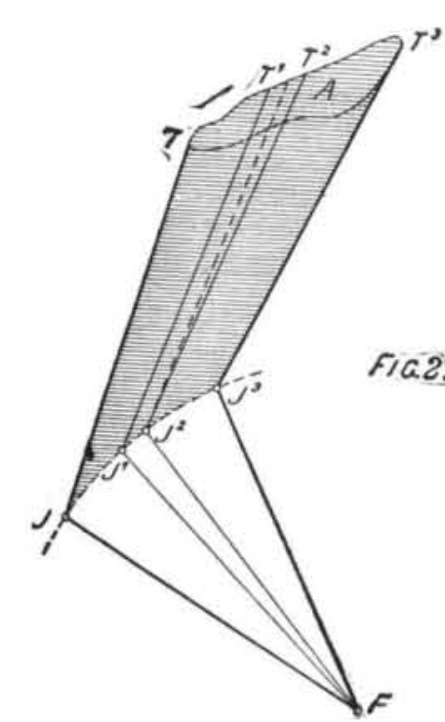

he instrument being shown, which, in distinction to light lines.

In order now to investigate what takes place durin the first part, represented in Fig. 2, we will consider the movement of the arm from the intermediate position,
$\mathrm{T}^{1} \mathrm{I}^{1}$, into an immediately adjoining one, $\mathrm{T}^{2} \mathrm{I}^{2}$. This progressive one, the direction of which is perpendicular o the arm and by means of which it arrives at the dotted position parallel to $\mathrm{T}^{1} \mathrm{I}^{1}$ and a rotating one, by into the actual new position, $\mathbf{T}^{2} \mathrm{I}^{3}$. If now we assume

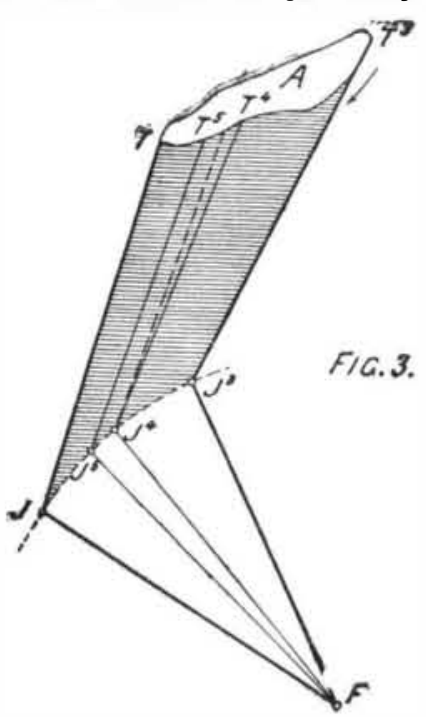

direction of the arrow, and that during the entire voyage of the arm from one extreme position into the other in the opposite direction, then we perceive that the shaded area in Fig. 2 can be expressed as the sum of the inclosed between two successive positions can be re-
solved. When it is further considered that the first solved. When it is further considered that the first movement of the arm, during which the space of the
parallelogram is covered, is at right angles to it, then it parallelogram is covered, is at right angles to it, then it
appears clear that during such movement the wheel wust roll off an arc, the length, $r$, of which is equal to ter is therefore equal to $\mathrm{M} r, \mathrm{M}$ being the length of the movable arm. We have accordingly :
W

Shaded area Fig. $2=\mathrm{M} \times$ sum of the various $r+$ The same line of argument will lead to a similar equathe shaded area in Fig. 3 , and as the difference between these two shaded areas is equal to the area in
closed by the curve, we obtain an expression for this latter by subtracting the second equation from the first one. This expression will be made up of two terms,
one containing the parallelograms and the other con one containing the parallelograms and the other con-
taining the sectors. In regard to the last term, it is clear that the sum of
In

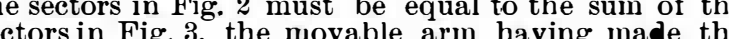
ectors in Fig. 3 , the movable arm having made the ors therefore annul each other. It is equally clear that the length rolled off by the wheel during the rotatory
movements inust be the same in both cases, the sum of novements inust be the same in both cases, the sum of Fig. 3 , and as their lengths are rolled off in Fig. 2 as in
ingite di rections, they will also annul each other; or in othe ing the sectors, the wheel at the end of its journey The term containing the sectors annulling itself, the xpression for the area inclosed by the curve is now

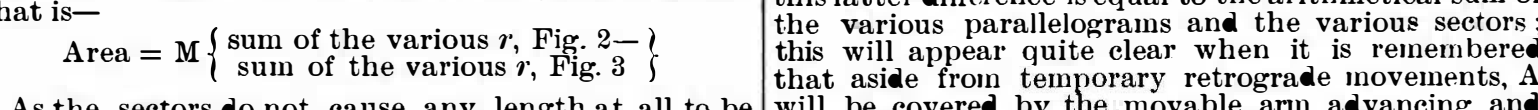
As the sectors do not cause any length at all to be
rolled off by the wheel, the difference in the brackets is
$\begin{aligned} & \text { the covered by the movable arm advancing and } \\ & \text { the wolling in a direction opposite to both mo }\end{aligned}$ clearly equal to the entire amount which the wheel
will be found to have rolled off on its return to the designating this amount with $R$

$$
\text { (1)............... Area }=\text { M R. }
$$

That is, the area inclosed by the curve is equal to the as a base and the length rolled off by the circumfernce of the wheel as its height.

In the above deduction the shape of the curve has been assumed to be such that the progressive motion and from the right to the left in Fig. 3 , is never interrupted by any temporary retrograde movement, at any
of the intermediate positions. This state of affairs, however, very rarely obtains in reality. The various
positions of the movable arm will, on the contrary, cross positions of the movable arm will, on the contrary, cross
and recross each other frequently, and the arm will often change the direction of its motion. But it is easy to prove that these complications
sult of our deductions in the least.

In each case of a retrograde movement of the movable arm, a portion of the wheel record will be annulled, such portion representing a certain portion of the
shaded area in either Fig. 2 or Fig. 3, according to first or in the second part of the journey. But it is clear that the movable arm will eventually have to pass over precisely the same area again, thus restoring the
annulled portion of the wheel record. All retrograde
movements will therefore cause certain amounts to movements will, therefore, cause certain amounts to
be added to, and precisely the same amounts to be subtracted from the shaded areas, as well as the whee for both. case the end, I, of the polar arm will describe a ful circle instead of an arc, as in the first case. Suppose now that this circle is inclosed entirely by the
curve itself, as shown in Fig. 4, then it is clear that the Fia. 4 .

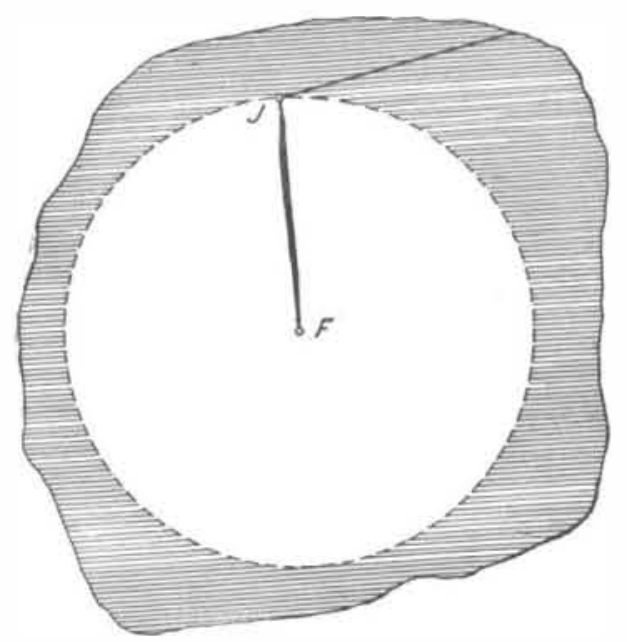

difference in area between the two (which difference i shaded in Fig. 4) must be equal to the sum of the vari ous parallelograms plus the sum of the various secjourney. As far as the sectors are concerned, nothing to have remained stationary during the occurrence of the various rotating movements, which carried th
arm from its first position through an entire circuit round $I$ as a center, back again to such first position. We perceive from this argument that the sum of the with the movable arm as a radius, that is,

Sum of various sectors $=M^{2} \pi$. roll off a length equal to the circumference of a cir-
cle the radius of which is its distance from I ; and desnating this with $l$, we have-

Wheel record corresponding to sectors $=2 l \pi$ The remaining part of the wheel record, according heights of the various small parallelograms.
Designating as before the entire wheel record with $\mathrm{R}$, we therefore obtain-

Sum of heights of parallelograms as given by wheel And further-

Sum of the various parallelograms $=M(R-2 l \pi)$.

The sum of the various parallelograms plus the sum d area, consequently-

Shaded area Fig. $4=M(R-2 l \pi)+M^{2} \pi$

And adding to this the contents of the circle described with the polar arm, $P$, as a radius, we clearly obtain

Entire area $=\mathrm{M}^{2} \pi+\mathrm{P}^{2} \pi+\mathrm{M}(\mathrm{R}-2 l \pi)$ or

(2) Entire area $=\mathrm{M} \mathrm{R}+\pi\left(\mathrm{M}^{2}+\mathrm{P}^{2}-2 \mathrm{M} l\right)$

The second term on the right side of this equation is a constant for each setting of the movable arm, the other rea is therefore found to be equal to a rectangle with the length of the movable arm as a base and the length height plus the circumference

height plus a certain constant. the circle described by the movable arm is not when entirely by the curve, as shown in Fig. 5. The difference between their areas in this case is equal to the ifference between the two shaded areas, $A$ and $B$, an
During this portion of the movement the wheel will 
tions while covering $B$. The difference of the area of the circle and the area of the curve will therefore be the various parallelograms and the various sectors, as in the foregoing case.

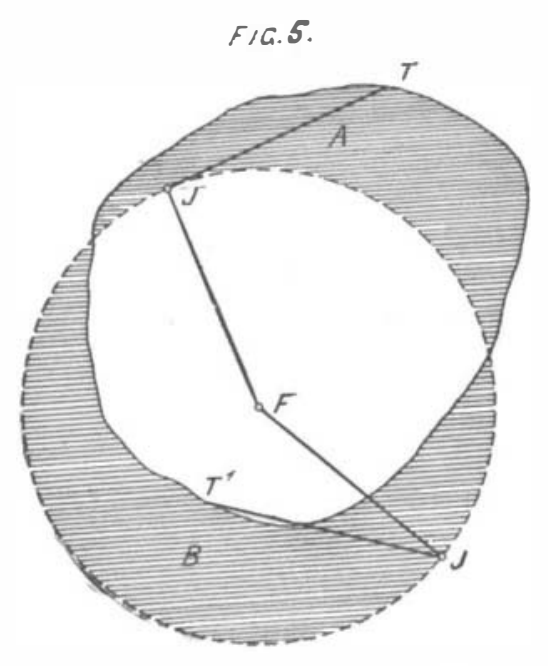

For practical use it is desirable that with the fulerum outside of the area the wheel record should render the latter direct, without the multiplication with the length of the movable arm being required. This can
be done by a proper adaptation of such length as be done

Suppose, for instance, the diameter of the wheel be $3 / 4^{\prime \prime}$, then the wheelrecord for $n$ revolutions is

$$
\mathrm{R}=n \frac{3}{4} \pi=2 \cdot 356 n
$$

and by substituting this into equation (1) we find: Area $=2.356 \mathrm{M} n$

In order now to let $n$ indicate the result directly, all that is required is to make the product $2 \cdot 356 \mathrm{M}$ equal
to either 1 or 10 or 100 or any other power of 10 . Supto either 1 or 10

$$
2 \cdot 356 \mathrm{M}=10, \text { then we have } \mathrm{M}=\frac{10}{2 \cdot 356}=4 \cdot 2
$$

That is, when the length of the movable arm is made cate an area of 10 square inches. The circumference of the wheel is graduated into 100 parts, and by means of a vernier the one thousandth part of one revolution just now, each vernier unit would correspond to the disk, conneted with the axle of the wheel by means of a worm, keeps a record of the number of revolutions up to ten. The reading to be taken at the colmmencement of a measurement therefore consists of four figures viz., the disk figure, the tenths and the hundredtbs of the wheel circumference, which are read on it direct,
and the thousandths, which are read by means of the vernier. Suppose that with the fulcrum outside of the area and with the above setting of the movable arm was 4269 , and that the reading taken after the tracer had traveled around the entire contour was 5374, then It is indifferent in which direction.

the tracer is watch travels, then the first reading has to be deducted from the second, and vice versa. In practice the above described method of obtaining the setting of the instrument, besides being too time-
robbing, is open to the objection that the requisite measurements cannot ordinarily be made with sufficient accuracy. The planimeters sold by the firm of by a table giving a limited number of settings, the graved on the movable arm. Any settings not contain ed in this table are found by trial, for which purpose a
trial disk is furnished, the grooved rim of which trial disk is furnished, the grooved rim of which
incloses a known area and around which the tracer incloses a known area and around which the tracer
can be carried with exactitude. The constant for inside positions of the fulcrum is figure such as a square or a circle, the contents of which can easily be found by computation. The differgiven by the planimeter, or more precisely the difference given by the planimeter, or more precisely the difference and that with the fulcrum inside, will be the outside the constant in the second case is positive or negative. If the latter, then the constant will of course be larger than either area or wheel record.

\section{IMPROVED PRIMARY BATTERY.}

By P. SELBY.

AMONG other things brought out here (Sydney, New South Wales) by me nearly four years ago was an electric pen apparatus with a Fuller bichromate bat tery. The dificulty of obtaining suitable cells and form of cell by which the expense and internal resistance of porous pots could be avoided. After sundry arrived at a stoneware jar containing the usual bichromate potass solution plus sulphuric acid, a piece of
thin platinum wire clothed with pure black India rubthin platinum wire elothed with pure black India rub-
ber, and a $1 / 8$ inch tube cemented to the wire at an ber, and a $1 / 8$ inch tube cemented to the wire at an
inch from the bottom. The spare inch of wire was then made into a flat spiral capahle of picking up a sulall quantity of mercury (Fig. 1), the India rubber tube being simply for insulation, so that the platinum jar of solution a good current was produced for a short time ; when withdrawn for examination, it was found vire, although action had ceased ; a fresh dip of mer-

cury restored the effect. On investigation I found that resistance of the fluid, but here it is different. Of the mercury had been used with zine, and was in fact
an amalgam. The absence of zine meant absence of
way would be to make the glass and the dish in one effect. On this result a simple and effective cell was (in glass), with piercings to insure the continuity of the weat jar) was divided off by a olass partition extaen te bichrom acreury in

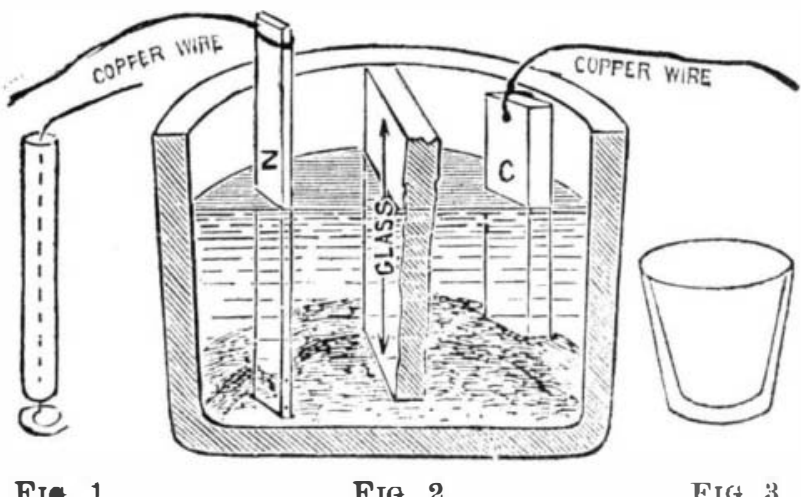

FIG. 2.

Fist. 3

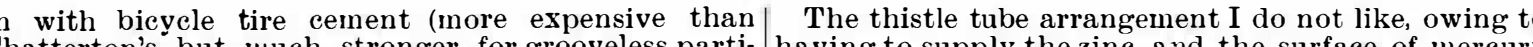
tions, jointing India rubber, gutta percha, shellac, and bitumen) reaching nearly to the bottom of jar ; mercury is then poured the fluids; carbon on the one side of the partition (but suspended so as not to touch the surface of the mer-
cury) in bichromate potass, sulphuric acid solution, and on the other side zine standing in the mercury in plain water to assist in counterbalancing the bichromate fluid on the other side, so as to require less mer-
cury to make a safe joint. Of course the jar is represented wider than is really the case, so as to prevent
confusion in the sketch. I was led to this form by many circumstances, among others the peculiar way mined "where it was outwardly protected by a cement which resisted the action of the dilute acid solution in the porous pot.

For cheapness and handiness, glass tumblers or drinking glasses with what are known as well bottoms
(Fig. 3) would make up very handy cells for experiFurther experiments led to a "thistle tube" of glass
nental purposes. Fent, the tube (Fig. 4) being bent in a gas
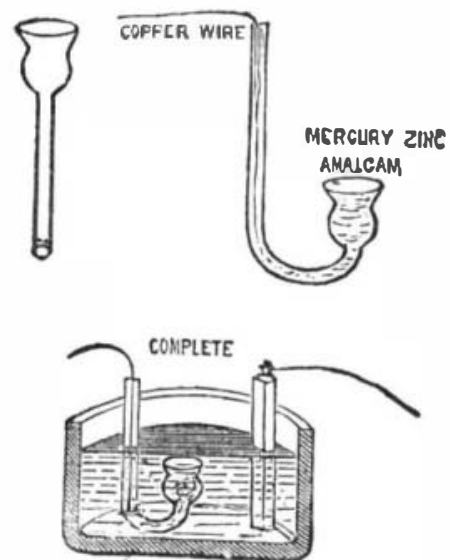

FIG. 4

[Concluded frøm SUPPLEMENT, No. 636, page 10160.]

HE POSITION AND PROSPECTS OF EIEC TRICITY AS APPIIED TO ENGIINEERING.* By William Geipel.

III-ELECTRIC LIGHTING.

OF the four branches of electric engineering dealt with in this paper, electric lighting is the one which up to the present has received most attention, called forth the largest outlay of capital, and produced the most beneficial results, if not to so great extent in this as in
other countries. Artificial illumination may be considered in the three aspects of comfort, convenience, and economy. As regards counfort, electric lighting proves itself superior to all other methods of illuminabe utilized and toned down to suit almost any require ment. It may be brought near to any object requirin llunination, without oceasioning the least inconin the ceilings or cornices, without risk of fire or of injury to the decoration. In short it can be used in any position or for any purpose of illumination for which gas, oil, or candles are available, and for a orea many for which they are not available. For outside illumination and large inclosures, the are light gives brilliancy and cheerfulness altogether unattainable by venience of the electric light has caused it to be highly appreciated, when it is found that by the nere press
ing of a button a light is instantly obtained, which can be shaded over in any manner, without danger of set-
ting fire to the fabrie forming the shade. It also does way with the constant cleaning of globes or trimmin of lamps. In respect of economy, the electric light does not as yet hold out the same decided advantages In incandeseent lighting the cost of distribution is still heavy though by increasing the electromotive force and the efficiency of lamps it is being much re duced. In arc lighting, the difficulty of subdividing and of reducing the amount of light given by one ar lamp renders it expensive for general outdoor street of umination, as compared with the present low price ame as shown, mercury placed therein, and copper of gas and oil. For the lighting of main streets and wire being passed down the stem into the mercury.
This is sinply placed in a jar of solution containing
light is required, the are light is, beyond question, far the carbon, but the mercury must receive occasional cheaper than gas, and its cost per candle power is but a replenishments of small pieces of sheet zine, which
very small fraction of that of gas. As the use of elec-
trie lighting extends, the cost of working becomes rereadily dissolve in it. The thistle tube is convenient tric lighting extends, the cost or worsing becomes respring clothes peg clipped on the top of the jar. But for permanent use a small shallow pot of earth5) has a round zine rod cemented in firmly, so as to

FI. $5 . \quad$ Fto. 0.
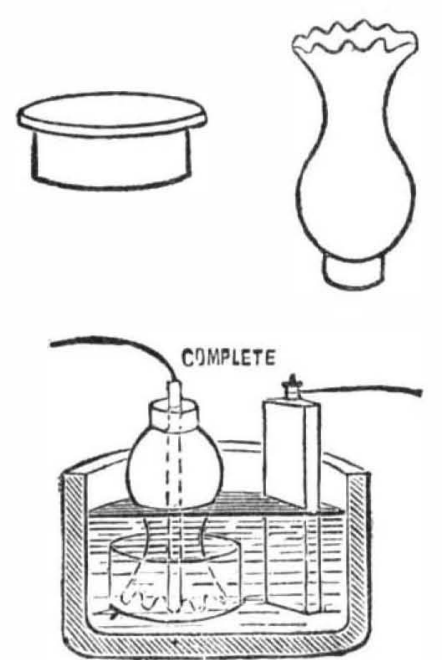

form a means of lifting in a complete state out of the outer battery jar, mercury is then poured in to form a "water joint," a para ffin lamp chimney with a "crink-
ed " top (Fig. 6) is then inverted and passed down the inc rod into the mercury and filled with pure water to (bichr. potass + ac. sulph., usual solution), in which stands the carbon.

Note. - This water remains purely free from any acid-
ity for months, and by careful tests I find that the addition of sulphuric acid simply weakens the batter by setting up a " counter" current. In the "porous are lamp per hour are now costing only $2 \mathrm{~d}$. The clie though the increased efficiency and durability of the apparatus have also greatly contributed to the reduc-
tion. Four years ago 11 nillimeter or 0.43 inch hard carbons cost $4 \mathrm{~d}$. per foot. They can now be obtained
for $11 / 4 \mathrm{~d}$. per foot, or less than one-third. The followfor $1 \frac{1}{4} \mathrm{~d}$. per foot, or less than one-third. The follow-
ing figures, supplied by the North British Railway, ing figures, supplied by the North British Railway,
respecting the actual cost of working their electric evey station, Edinburgh, are inte ing as showing how much cheaper it is becoming. The installation is worked by their own staff, and consists now of forty Brush are lamps, supplied with a current driven by a semi-fixed engine.

\begin{tabular}{|c|c|c|c|}
\hline & $£$ & s. & d. \\
\hline Wages. & 165 & 13 & \\
\hline Repairs...... & 47 & 2 & \\
\hline Carbons... & 125 & 15 & 11 \\
\hline Coal.. & 65 & 19 & \\
\hline Oil, stores, ete. & 27 & 15 & \\
\hline $\begin{array}{l}\text { Interest and depreciation at } 10 \text { per } \\
\text { cent } \ldots \ldots \\
\text { en. }\end{array}$ & 52 & 2 & 2 \\
\hline
\end{tabular}

July to December, 1884, Thirty-three Arc Lamps, 41,884

Equal to $2 \cdot 77$ d. per lamp hour. $\$ 484403$

July to December, 1886, Thirty-nine ArC Lamps, 55,068

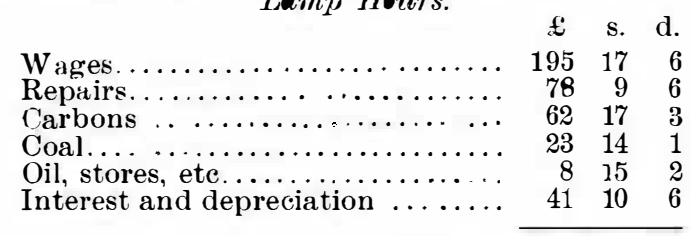

Equal to $1{ }^{\bullet} 79 d$. per lamp hour. $\$ 411 \quad 4 \quad 0$ In conjunction with these are lamps, they are run-
ing 148 Brush Victoria incandescent lamps, distributed *A A paper recently read before the Institution of Mechanical Enginecrey,
London. 